\title{
A proposal to revise the disruption index
}

\author{
Loet Leydesdorff; Alexander Tekles; Lutz Bornmann
}

How to cite this article:

Leydesdorff, Loet; Tekles, Alexander; Bornmann, Lutz (2021). "A proposal to revise the disruption index". Profesional de la información, v. 30, n. 1, e300121.

https://doi.org/10.3145/epi.2021.ene.21

Letter received on February, $21^{\text {st }} 2020$

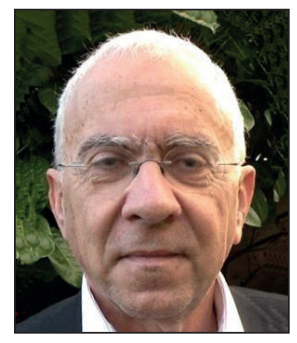

Loet Leydesdorff

https://orcid.org/0000-0002-7835-3098

University of Amsterdam

Amsterdam School of Communication

Research (ASCoR)

PO Box 15793

1001 NG Amsterdam, The Netherlands

loet@leydesdorff.net

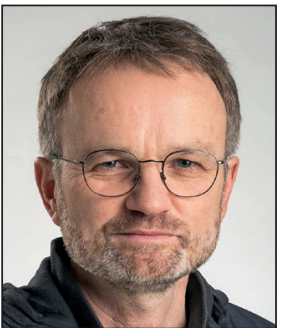

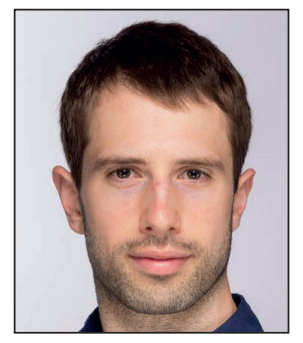

Alexander Tekles https://orcid.org/0000-0001-8765-9331

Administrative Headquarters of the Max Planck Society

Science Policy and Strategy Department alexander.tekles.extern@gv.mpg.de

Ludwig-Maximilians-University Munich, Department of Sociology

\section{Abstract}

The disruption index $(D I)$ based on bibliographic coupling and uncoupling between a document and its references was first proposed by Funk \& Owen-Smith (2017) for citation relations among patents and then adapted for scholarly papers by Wu et al. (2019). However, Wu \& Wu (2019) argued that this indicator would be inconsistent. We propose revised disruption indices $\left(D I^{*}\right.$ and $\left.D I^{*}\right)$ which make the indicator theoretically more robust and consistent. Along similar lines, Chen et al. (2020) developed the indicator into two dimensions: disruption and consolidation. We elaborate the improvements in simulations and empirically. The relations between disruption, consolidation, and bibliographic coupling are further specified. Bibliographic coupling of a focal paper with its cited references generates historical continuity. A two-dimensional framework is used to conceptualize discontinuity not as a residual, but a dimension which can further be specified.

\section{Keywords}

Disruption; Consolidation; Indicator; Revision; Bibliographic coupling.

\section{Introduction}

Wu et al. (2019) introduced the Disruption index (DI) for scholarly citation data, in analogy to the $C D$ index proposed by Funk \& Owen-Smith (2017) for studying patent citations. In the latter paper a seemingly complex formula for disruption is provided, as follows:

$$
C D_{t}=\frac{1}{n} \sum_{i=1}^{n} \frac{-2 f_{i t} b_{i t}+f_{i t}}{w_{i t}}, w_{i t}>0
$$

where

$$
f_{i t}= \begin{cases}1 & \text { if } i \text { cites the focal patent } \\ 0 & \text { otherwise }\end{cases}
$$

and

$$
b_{i t}= \begin{cases}1 & \text { if } i \text { cites a reference of the focal patent } \\ 0 & \text { otherwise }\end{cases}
$$


Wu et al. (2019) rewrote Eq. 1 in a simpler form as follows:

$$
D I=\frac{N_{F}-N_{B}}{N_{F}+N_{B}+N_{R}}
$$

where

- $N_{B}=$ Number of papers citing both the focal paper (FP) and at least one of its references; the citing papers couple the focal paper bibliographically with its references and would thus indicate "continuity" since they span two "generations" of papers in terms of citations;

- $N_{F}=$ Number of papers citing exclusively the FP and not one of its references; this set is considered "disruptive" because the citations do not reach historically back to papers cited by FP;

- $N_{R}=$ Number of papers citing references of FP, but not FP itself.

In other words: documents (patents or papers) can be coupled bibliographically to their own cited references by citing papers (Kessler, 1963) and thus indicate historical continuity across "generations" of citations. Alternatively, papers can be unrelated to previously cited research. The generation of continuity by bibliographic coupling can also be considered as a sign of "consolidation" (Chen et al., 2020). In Eq. 4, for example, $N_{F}=0$ and $N_{R}=0$ with $N_{B}>0$ would mean $D I=-N_{B} / N_{B}=-1$ and thus extreme consolidation; vice versa, $N_{B}=0$ and $N_{R}=0$, while $N_{F}>0$ leads to $D I=1$ and thus extreme disruption. In this model, however, disruption and consolidation are traded-off on a single dimension, while one can also model the two concepts as two independent dimensions.

In the numerator of Eq. $4\left(N_{F}-N_{B}\right)$, the number of papers bibliographically coupling FP with FP's references $\left(N_{B}\right)$ is subtracted from $N_{F}$. The difference between the total number of citing papers $\left(N_{F}+N_{B}\right)$ and the value in the numerator of Eq. 4 is $\left(N_{F}+N_{B}\right)-\left(N_{F}-N_{B}\right)=2 * N_{B}{ }^{1}$ One could argue that it would be more parsimonious to subtract $N_{B}$ only once from the total citations $\left(N_{F}+N_{B}\right)$. One then obtains an indicator $D I^{*}$ for the disruption, which can be formulated as follows:

$$
D I^{*}=\frac{N_{F}}{N_{F}+N_{B}+N_{R}}
$$

Analogously, one can define an indicator for consolidation $D I^{\#}$ as follows:

$$
D I^{\#}=\frac{N_{B}}{N_{F}+N_{B}+N_{R}}
$$

$D I^{*}$ is a measure of disruption and $D I^{*}$ a measure of consolidation.

Wu \& Wu (2019) noted that $N_{F}-N_{B}$ can be negative when most of the citations of FP couple FP bibliographically to its references. This leads to a negative value of $D /$ between minus one and zero, indicating that "continuity" prevails as the opposite of disruption. Increases in the value of $N_{R}$ (other references), however, lead to less disruption if $N_{F}-N_{B}>0$, but enhance disruption when $N_{F}-N_{B}<0$. Wu \& Wu (2019) considered this effect as "inconsistent," and called for a revision of the indicator. Using the absolute values, however, $N_{F}-N_{B}$ and $N_{B}-N_{F}$ can be measures of both disruption and continuity. The problem can be solved by using $D I^{*}$ or $D I^{\#}$ as independent indicators in a two-dimensional model.

\section{2. $D I_{n}:$ a further extension}

Bornmann et al. (2020) and Bornmann \& Tekles, (2020) extended DI to $D I_{n^{\prime}}$, where $n$ denotes the threshold value for counting the bibliographic couplings between FP and its references in a single citing paper. Only papers which cite $n$ or more references among the cited references of FP are counted in $M_{B}^{n}$, which is used in Eq. 4 instead of $N_{B}$. It follows that the original indicator is the same as $D I_{1}$. The problem in the background is that $D I$ tends to indicate many papers as close to zero. By adding thresholds, the authors aimed to adjust the indicator in order to focus the indicator on identifying disruptive research (Bornmann et al., 2020).

A disadvantage of this computational strategy can be that the number of possible indicators proliferates into "families" of indicators (Bu et al., in press). For example, one can replace $N_{\mathrm{B}}$ with $\boldsymbol{M}_{B}^{n}$ in both the numerator and the denominator (Eq. 7) or only in the numerator and keep the denominator constant (Eq. 8).

$$
\begin{aligned}
& D I_{n}^{a}=\left(N_{F}-M_{B}^{n}\right) /\left(N_{F}+M_{B}^{n}+N_{R}\right) \\
& D I_{n}^{b}=\left(N_{F}-M_{B}^{n}\right) /\left(N_{F}+N_{B}+N_{R}\right)
\end{aligned}
$$

Bornmann et al. (2020) used Eq. 7. In Eq. 7, the replacement of $N_{B}$ has an effect on both the numerator and the denominator. In the case of Eq. 8, one keeps the domain in the denominator the same between $D I$ and $D I_{n}$. One can thus compare among proportions. 


\section{An extreme example}

An extreme and counter-intuitive case, for example, can be formulated as follows:

Consider two papers A and B. For paper A: $N_{F}=10, N_{B}=10$, and $N_{R}=100$; for paper B: $N_{F}=10, N_{B}=100$, and $N_{R}$ = 10 (see Table 1$)$.

Do these two papers have the same disruption or not? Table 1 explicates the computation for the two papers thus specified. Paper A scores $D I=0.0$. While $D I=-0.75$ for paper $B-$ an increase of 75 percentage points in the continuity- $D I *$ is 0.083 for both papers. DI* indicates that the papers have the same level of disruption; DI shows a different level of disruption. However, since $D I$ is $\leq 0$ for paper $A$, the difference between the papers is on the consolidation rather than the disruption dimension: both papers are consolidating, but paper B is more consolidating than paper $A$. This can also be seen when using $D I^{*}$ and $D I^{*}$ : the two papers have the same levels of disruption $\left(D I^{*}\right)$, while the level of consolidation $\left(D I^{\#}\right)$ is different.

The two effects - changes in the values of $N_{B}$ or $N_{R}$ - are combined and perhaps confusing in this example. However, one can distinguish the two effects - disruption and consolidationanalytically by using simulations. The ten-times larger value of $N_{B}$ in the second paper leads to a ten times larger consolidation $\left(D I^{\sharp}\right)$.

\section{Simulations}

Let us, for example, focus on the effects of increasing $N_{B}$. Given an initial configuration with $N_{F}=20, N_{B}=10$, and $N_{R}=20$, we assume the addition of a single citation to $N_{B}$ at each step from ten to one hundred (Table 2). This makes $D I$ increasingly negative. In other words, the continuity increases and $D I$ decreases. The numerator of $D I^{\#}$ increases, while $D I^{*}$ decreases because of the increase of the denominator. Figure 1 shows the respective curves in the case of a stepwise increase of $N_{B}$ from ten to one hundred for $D I, D I^{*}$, and $D I^{\#}$.

Both $D I^{*}$ and $D l^{\#}$ are by definition positive. As $N_{B}$ and consequently the bibliographic coupling and $D l^{\#}$ increase, this seems to be the consolidation indicator, whereas $D I^{*}$ is the disruption indicator. As noted, $D I$ itself is complex since it combines disruption and consolidation in a single dimension.

\section{Empirical examples}

We compare the results for two empirical cases. In the first case, we use the set of Bornmann \& Tekles (2019): that is, 566 papers published in Scientometrics between 2000 and 2010 with at least 10 citations and 10 cited references each (Bornmann et al., 2020). Table 4 shows the top-20 lists for $D I, D I^{*}, D I^{\#}$, and $D I_{5}$. The paper by Heinze et al. (2007) is listed at the first position in three of the four lists in Table 4; followed by Glänzel et al. (2003) in two of the four lists. However, Glänzel et al. (2003) is only on the tenth position using $D I$ for the measure-

Table 1. $D I, D I^{*}$, and $D I^{\#}$ for an extreme case

\begin{tabular}{|l|l|c|c|}
\cline { 3 - 4 } \multicolumn{2}{c|}{} & A & B \\
\hline & $N_{F}$ & 10 & 10 \\
\hline & $N_{B}$ & 10 & 100 \\
\hline & $N_{R}$ & 100 & 10 \\
\hline$D I$ & & & -90 \\
\hline & Numerator & 0 & 120 \\
\hline & Denominator & 120 & -0.75 \\
\hline$D I^{*}$ & DI & 0 & 10 \\
\hline & & 10 & 120 \\
\hline & Numerator & 120 & 0.083 \\
\hline$D I^{*}$ & Denominator & 0.083 & 100 \\
\hline & $D I^{*}$ & 10 & 120 \\
\hline & Numerator & 120 & 0.83 \\
\hline & Denominator & 0.083 & \\
\hline & DI & & \\
\hline
\end{tabular}

Table 2. Simulation based on increasing values for $N_{\mathrm{B}}$, while holding $N_{\mathrm{F}}$ and $N_{\mathrm{R}}$ constant

\begin{tabular}{|c|c|c|c|c|c|}
\hline $\boldsymbol{N}(\boldsymbol{B})$ & $\boldsymbol{N}(\boldsymbol{F})$ & $\boldsymbol{N}(\boldsymbol{R})$ & $\boldsymbol{D}$ & $\boldsymbol{D I}^{*}$ & $\boldsymbol{D I}^{*}$ \\
\hline 10 & 20 & 20 & 0.200 & 0.400 & 0.200 \\
\hline 11 & 20 & 20 & 0.176 & 0.392 & 0.216 \\
\hline 12 & 20 & 20 & 0.154 & 0.385 & 0.231 \\
\hline 13 & 20 & 20 & 0.132 & 0.377 & 0.245 \\
\hline 14 & 20 & 20 & 0.111 & 0.370 & 0.259 \\
\hline 15 & 20 & 20 & 0.091 & 0.364 & 0.273 \\
\hline 16 & 20 & 20 & 0.071 & 0.357 & 0.286 \\
\hline 17 & 20 & 20 & 0.053 & 0.351 & 0.298 \\
\hline 18 & 20 & 20 & 0.034 & 0.345 & 0.310 \\
\hline$\ldots$ & $\ldots$ & $\ldots$ & $\ldots$ & $\ldots$ & $\ldots$ \\
\hline 100 & 20 & 20 & -0.600 & 0.133 & 0.733 \\
\hline
\end{tabular}

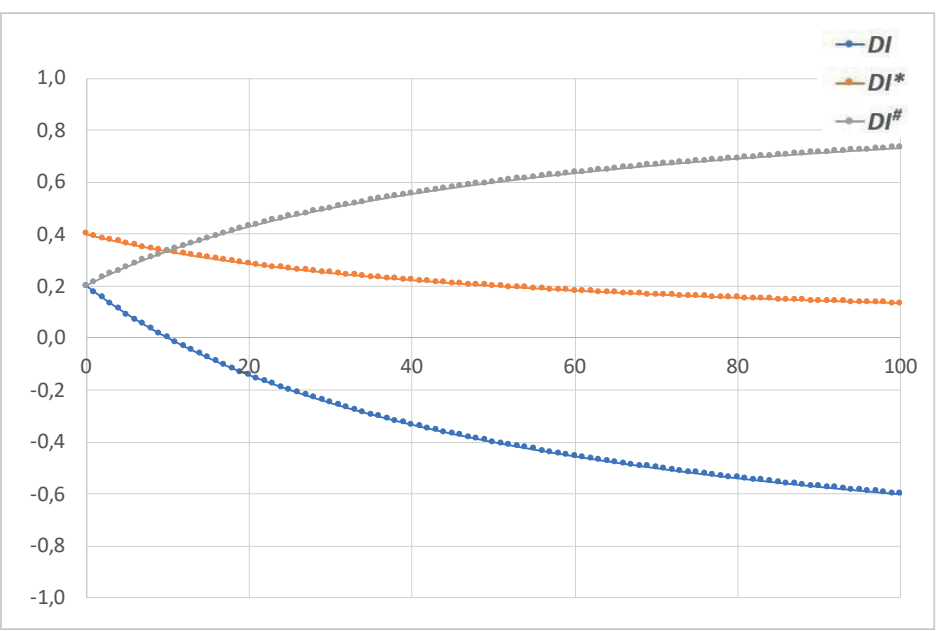

Figure 1. Development of $D I, D I^{*}$, and $D I^{*}$ with increasing values of $N_{\mathrm{B}}$ 
ment. Both papers focus on the institutional conditions of creativity, novelty production, and disruption. In our opinion, since both papers are a bit programmatic, high disruption values can be expected.

Table 4. Rank-ordering of the top-20 papers in the sample of 566 papers published between 2000 and 2010 in Scientometrics using $D I, D I^{*}, D l^{*}$, and $D I_{5}$.

\begin{tabular}{|c|c|c|c|c|c|c|c|}
\hline \multicolumn{2}{|r|}{ DI } & \multicolumn{2}{|r|}{$D I^{*}$} & \multicolumn{2}{|r|}{ Dli $^{*}$} & \multicolumn{2}{|r|}{$\mathrm{DI}_{5}$} \\
\hline Heinze T, 2007, V70, P125 & 0.182 & Heinze T, 2007, V70, P125 & 0.205 & Glänzel W, 2003, V58, P571 & 0.125 & Heinze T, 2007, V70, P125 & 0.205 \\
\hline Bordons M, 2002, V53, P195 & 0. & inzel W, 2003, V58, P571 & 71 & Bar-llan J, 2004, V59, P391 & 117 & Glänzel W, 2003, V58, P571 & 0.171 \\
\hline Chiu WT, 2004, V61, P69 & 0.107 & Bordons M, 2002, V53, P195 & 165 & Burrell QL, 2005, V65, P381 & 0.109 & Bordons M, 2002, V53, P195 & .165 \\
\hline Patra SK, 2006, V67, P477 & 06 & Archambault E, 2006, V68, P329 & 0.149 & Prpic K, 2002, V55, P27 & 102 & Archambault E, 2006, V68, P329 & 0.149 \\
\hline Boshoff N, 2009, V81, P413 & 0.064 & Glänzel W, 2002, V53, P171 & 0.116 & Archambault E, 2006, V68, P329 & 0.101 & Glänzel W, 2002, V53, P171 & 0.116 \\
\hline Van Eck NJ, 2010, V84, P523 & 0.063 & Van Eck N. & 14 & Schloegl C, 2010, V82, & 093 & Van Eck & 0.114 \\
\hline Glänzel W, 2002, & 3 & 9 & 14 & 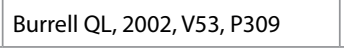 & 889 & $04, V 61, P 69$ & 114 \\
\hline Van Raan AFJ, 2005, V62, P133 & 56 & ra SK, & 06 & rter AL, & 088 & tra SK, 2006, V67, P477 & 0.106 \\
\hline Archambault E, 2006, V68, P329 & 0.047 & n Raan AFJ, 2005, V62, P133 & 0.094 & Burrell QL, 2001, V52, P3 & 0.073 & Van Raan AFJ, 2005, V62, P133 & 0.094 \\
\hline Glänzel W, 2003, V58, P571 & 0.046 & Ren SL, 2002, V53, P389 & 0.093 & Schummer J, 2004, V59, P425 & 0.070 & Ren SL, 2002, V53, P389 & 0.093 \\
\hline Weingart P, 2005, V62, P117 & 0.044 & Nederhof AJ, 2006, V66, P81 & 0.079 & Uzun A, 2004, V61, P457 & 0.068 & Nederhof AJ, 2006, V66, P81 & 0.079 \\
\hline Keiser J, & 0. & Boshoff N, 2009, V81, P413 & 0.079 & Hagen $\mathrm{I}$ & 0.067 & Boshoff N, 2009, V81, P413 & 0.079 \\
\hline Hsieh WH, 20 & 0 & 93 & & 9 & 66 & 293 & 0.076 \\
\hline Rinia EJ, 2001, V51, P293 & 8 & Weingart $P, 2005$, V62, P117 & 71 & Boyack KW, 2005, V64, P351 & 066 & eingart $P, 2005, V 62, P 117$ & 0.071 \\
\hline Glänzel W, 2006, V67, P67 & 0.036 & Porter AL, 2007, V72, & 0.067 & Vaughan L, 2006, V67, P291 & 0.065 & Porter AL, 2007, V72, P117 & 0.067 \\
\hline Liu CY, 2010, V82, P21 & 0.034 & Glänzel W, 2006, V67, P67 & 0.062 & Nederhof AJ, 2006, V66, & 0.064 & Glänzel W, 200 & 0.062 \\
\hline Lars & 0 & $\mathrm{BC}$ & 99 & L & 53 & 51 & 59 \\
\hline Tijssen RJW, 2002, V54, P381 & 4 & Keiser J, 2005, V62, P351 & 88 & Bordons M, 2003, V57, P159 & 0.063 & eiser J, 2005, V62, P351 & 0.058 \\
\hline Ren SL, 2002, V53, P389 & 0.032 & Bornmann L, 2006, V68, P427 & 0.058 & Ren SL, 2002, V53, P389 & 0.061 & Bornmann L, 2006, V68, P427 & 0.058 \\
\hline Lewison G, 2001, V52, P29 & 0.029 & Tijssen RJW, 2002, V54, P381 & 0.057 & Glänzel W, 2002, V53, P171 & 0.054 & Tijssen RJW, 2002, V54, P381 & 0.057 \\
\hline
\end{tabular}

Table 5. Rank-ordering of the top-20 papers in the sample of 9,251 papers published between 2000 and 2010 in Nature using $D I, D I^{*}, D l^{*}$, and $D I_{5}$.

\begin{tabular}{|c|c|c|c|c|c|c|c|}
\hline & DI & & $D I^{*}$ & & $D I^{*}$ & & $\mathrm{DI}_{5}$ \\
\hline Chen G, 2000, V407, P361 & 0.609 & Chen G, 2000, V407, P361 & 0.670 & Tegus O, 2002, V415, P150 & 0.346 & Chen G, 2000, V407, P361 & 0.669 \\
\hline Myers N, 2000, V403, P853 & 0.543 & Myers N, 2000, V403, P853 & 0.607 & Aoki D, 2001, V413, P613 & 0.285 & Myers N, 2000, V403, P853 & 0.605 \\
\hline Poizot P, 2000, V407, P496 & 0.523 & Poizot P, 2000, V407, P496 & 0.598 & Calvi L, 2003, V425, P841 & 0.244 & Poizot P, 2000, V407, P496 & 0.598 \\
\hline Erlebacher J, 2001, V410, P450 & 0.347 & Erlebacher J, 2001, V410, P450 & 0.481 & Khriachtchev L, 2000, V406, P874 & 0.238 & Erlebacher J, 2001, V410, P450 & 0.477 \\
\hline Bertotti B, 2003, V425, P374 & 0.325 & Bertotti B, 2003, V425, P374 & 0.419 & Parish M, 2003, V426, P162 & 0.236 & Bertotti B, 2003, V425, P374 & 0.419 \\
\hline Saito Y, 2004, V432, P84 & 0.310 & Forterre Y, 2005, V433, P421 & 0.395 & Stone EC, 2008, V454, P71 & 0.229 & Forterre Y, 2005, V433, P421 & 0.392 \\
\hline Forterre Y, 2005, V433, P421 & 0.300 & Ernst M, 2002, V415, P429 & 0.379 & Day J, 2007, V450, P853 & 0.224 & Ernst M, 2002, V415, P429 & 0.378 \\
\hline White S, 2001, V409, P794 & 0.270 & Saito Y, 2004, V432, P84 & 0.373 & Rong H, 2005, V433, P725 & 0.223 & Saito Y, 2004, V432, P84 & 0.372 \\
\hline Marescaux J, 2001, V413, P379 & 0.257 & Strukov DB, 2008, V453, P80 & 0.338 & Barland S, 2002, V419, P699 & 0.223 & White S, 2001, V409, P794 & 0.334 \\
\hline Strukov DB, 2008, V453, P80 & 0.236 & White S, 2001, V409, P794 & 0.337 & Tschop M, 2000, V407, P908 & 0.221 & Strukov DB, 2008, V453, P80 & 0.334 \\
\hline Margulies M, 2005, V437, P376 & 0.231 & Marescaux J, 2001, V413, P379 & 0.319 & Coles H, 2005, V436, P997 & 0.217 & Marescaux J, 2001, V413, P379 & 0.315 \\
\hline Moss RH, 2010, V463, P747 & 0.227 & James C, 2005, V434, P1144 & 0.309 & Porath D, 2000, V403, P635 & 0.216 & Steele B, 2001, V414, P345 & 0.302 \\
\hline Steele B, 2001, V414, P345 & 0.226 & Greffet J, 2002, V416, P61 & 0.306 & Loll B, 2005, V438, P1040 & 0.213 & James C, 2005, V434, P1144 & 0.301 \\
\hline Magurran A, 2003, V422, P714 & 0.225 & Steele B, 2001, V414, P345 & 0.302 & Niemela J, 2000, V404, P837 & 0.202 & Greffet J, 2002, V416, P61 & 0.301 \\
\hline Rost S, 2004, V427, P537 & 0.216 & Rost S, 2004, V427, P537 & 0.298 & Donnelly C, 2006, V439, P843 & 0.201 & Rost S, 2004, V427, P537 & 0.294 \\
\hline Ernst M, 2002, V415, P429 & 0.212 & Tomita M, 2003, V421, P517 & 0.291 & Ritz T, 2004, V429, P177 & 0.197 & Armand M, 2008, V451, P652 & 0.290 \\
\hline Cortright R, 2002, V418, P964 & 0.211 & Gomes R, 2005, V435, P466 & 0.291 & Takasaki T, 2000, V403, P913 & 0.193 & Tomita M, 2003, V421, P517 & 0.287 \\
\hline Davies H, 2002, V417, P949 & 0.208 & Armand M, 2008, V451, P652 & 0.290 & Greiner M, 2002, V415, P39 & 0.192 & Gomes R, 2005, V435, P466 & 0.284 \\
\hline Day P, 2003, V425, P817 & 0.207 & Day P, 2003, V425, P817 & 0.277 & Shelly DR, 2007, V446, P305 & 0.192 & Magurran A, 2003, V422, P714 & 0.274 \\
\hline Reibold M, 2006, V444, P286 & 0.203 & Corma A, 2001, V412, P423 & 0.275 & Hu D, 2009, V461, P640 & 0.191 & Day P, 2003, V425, P817 & 0.273 \\
\hline
\end{tabular}

In the second example, we selected Nature papers with at least 10 citations and 10 cited references each (with $N=9,251$ papers). Table 5 shows the top-20 lists for $D I, D I^{*}, D I^{\#}$, and $D I_{5}$. The comparison of Table 5 with Table 4 reveals that the disruption values in Table 5 are significantly higher than the values in Table 4: the highest values in Table 4 are on the 
level as the lowest values in Table 5. The comparably high values for Nature papers are expectable, since it is the mission of the journal to publish the

"finest peer-reviewed research in all fields of science and technology on the basis of its originality, importance, interdisciplinary interest, timeliness, accessibility, elegance and surprising conclusions" https://www.nature.com/nature/about

Table 6a (columns a to d). Correlations among $D I, D I^{*}, D I^{\#}$, and $D I_{5}$ in the study of 566 papers in Scientometrics during the period 2000-2010. Lower triangle: Spearman rank-order correlations; upper triangle: Pearson correlations. All correlations are statistically significant at the $1 \%$-level.

Table $6 \mathrm{~b}$ (columns $\mathrm{f}$ to i). Correlations among $D I, D I^{*}, D I^{*}$, and $D I_{5}$ in the study of 9,251 papers in Nature during the period 2000-2010. Lower triangle: Spearman rank-order correlations; upper triangle: Pearson correlations. All correlations are statistically significant at the $1 \%-l e v e l$.

\begin{tabular}{|c|c|c|c|c|c|c|c|c|}
\hline \multicolumn{5}{|c|}{ Correlations Scientometrics } & \multicolumn{4}{|c|}{ Correlations Nature } \\
\hline $\begin{array}{l}\text { DI } \\
\text { (a) }\end{array}$ & $\begin{array}{l}D I^{*} \\
\text { (b) }\end{array}$ & $\begin{array}{l}D I^{\#} \\
(c)\end{array}$ & $\begin{array}{l}D I_{5} \\
\text { (d) }\end{array}$ & (e) & $\begin{array}{l}D I \\
\text { (f) }\end{array}$ & $\begin{array}{c}D I^{*} \\
(\mathrm{~g})\end{array}$ & $\begin{array}{l}\boldsymbol{D I}^{\#} \\
\text { (h) }\end{array}$ & $\begin{array}{c}\mathrm{DI}_{5} \\
\text { (i) }\end{array}$ \\
\hline 1 & .636 & -.301 & 699 & DI & 1 & .525 & -.408 & .636 \\
\hline .217 & 1 & .544 & .986 & $D I^{*}$ & .525 & 1 & .563 & .976 \\
\hline-.443 & .680 & 1 & .458 & $D I^{\#}$ & -.408 & .563 & 1 & .429 \\
\hline .386 & .918 & .485 & 1 & $D I_{5}$ & .636 & .976 & .429 & 1 \\
\hline
\end{tabular}

These correlation matrices are not so different when compared among both tables. For example, $D I^{\#}$ is always negatively correlated with $D I$. Unlike $D I, D i^{*}$, and $D I_{5}, D I^{*}$ is exclusively an indicator of consolidation, whereas $D I^{*}$ is a disruption indicator. Despite the high correlations between $D I^{*}$ and $D I(r>0.9)$, the zero-hypothesis that the median of these two distributions is the same, is rejected using the Related Samples Wilcoxon Signed Rank Test at the 5\% level. None of the $D I^{\#}$ top papers occurs in the top papers for the other indicators and vice versa.

\section{Conclusions and discussion}

Following Chen et al. (2020), we have taken the two dimensions of disruption and continuity or consolidation apart. DI can be considered as a continuity indicator more than a disruption indicator since the operation is grounded in bibliographic coupling. The bibliographic coupling of a focal paper to its references generates a representation of continuity. From this perspective, discontinuity is indicated when the bibliographic coupling is not sufficiently generating continuity. This is analogous to the graphical representations that one can make with programs such as HistCite ${ }^{\mathrm{TM}}$ (Garfield et al., 2003) or CitNetExplorer (Van Eck; Waltman, 2014). One sees the lines of continuity along trajectories. Discontinuities are inferred where the lines are interrupted. However, the semantics of using two words for a single indicator with opposite sign can be confusing.

In our opinion, the choice for parameters should be legitimated by theoretical reasoning. The subtraction of $N_{\mathrm{B}}$ for a second time, for example, may not be necessary to detect disruptive papers and - as shown by $\mathbf{W u} \& \mathbf{W u}(2019)$ - can lead to confusion in the results. The revised indicators $D I^{*}$ and $D I^{\#}$ solve these problems and simplify both the computation and the semantics.

It may appear that this issue concerns a detail, since in many cases the values of $D I^{*}$ and $D I$ will be approximately the same. For analytical reasons, however, $D I^{*} \geq D I$. DI adds to bibliographic coupling - a theoretical instrument in bibliometrics - by focusing specifically on the couplings and un-couplings between a paper and its references. In a follow-up paper, we envisage to discuss disruption in relation to critical transitions in a time-series of events (cf. Leydesdorff $e t$ al. 2018; Leydesdorff, 1991). Both measures (disruption and critical transition) seek to analyze change at the level of the system. While preparing that paper, we stumbled into the problems of semantics and operationalization that we hope to have clarified with this more methodologically oriented paper.

\section{Note}

1. This value explains the number two in Eq. 1 which can be made more visible by rewriting the equation as follows:

$$
C D_{t}=\frac{1}{n} \sum_{i=1}^{n} \frac{f_{i t}\left(1-2 b_{i t}\right)}{w_{i t}}, w_{i t}>0
$$

\section{References}

Bornmann, Lutz; Devarakonda, Sitaram; Tekles, Alexander; Chacko, George (2020). "Disruptive papers published in Scientometrics: Meaningful results by using an improved variant of the disruption index originally proposed by Wu, Wang, and Evans (2019)". Scientometrics, v. 123, pp. 1149-1155.

https://doi.org/10.1007/s11192-020-03406-8

Bornmann, Lutz; Tekles, Alexander (2019). "Disruptive papers published in Scientometrics". Scientometrics, v. 120, pp. 331-336.

https://doi.org/10.1007/s11192-019-03113-z 
Bornmann, Lutz; Tekles, Alexander (2020). "Convergent validity of several indicators measuring disruptiveness with milestone assignments to physics papers by experts", arXiv:2006.10606.

https://arxiv.org/abs/2006.10606

Bu, Yi; Waltman, Ludo; Huang, Yong (in press). "A multidimensional framework for characterizing the citation impact of scientific publications". Quantitative science studies.

https://www.mitpressjournals.org/doi/abs/10.1162/qss_a_00109

Chen, Jiyao; Shao, Diana; Fan, Shaokun (2020). "Destabilization and consolidation: Conceptualizing, measuring, and validating the dual characteristics of technology". Research policy, v. 50, n. 1, art. 104115.

https://doi.org/10.1016/j.respol.2020.104115

Funk, Russell J.; Owen-Smith, Jason (2017). “A dynamic network measure of technological change”. Management science, v. 63, n. 3, pp. 791-817.

https://doi.org/10.1287/mnsc.2015.2366

Garfield, Eugene; Pudovkin, Alexander I.; Istomin, Vladimir S. (2003). "Why do we need algorithmic historiography?". Journal of the American Society for Information Science and Technology, v. 54, n. 5, pp. 400-412.

https://doi.org/10.1002/asi.10226

Glänzel, Wolfgang; Schlemmer, Balázs; Thijs, Bart (2003). “Better late than never? On the chance to become highly cited only beyond the standard bibliometric time horizon". Scientometrics, v. 58, n. 3, pp. 571-586.

https://doi.org/10.1023/B:SCIE.0000006881.30700.ea

Heinze, Thomas; Shapira, Philip; Senker, Jacqueline; Kuhlmann, Stefan (2007). "Identifying creative research accomplishments: Methodology and results for nanotechnology and human genetics". Scientometrics, v. 70, n. 1, pp. 125-152. https://doi.org/10.1007/s11192-007-0108-6

Kessler, Myer-Mike (1963). "Bibliographic coupling between scientific papers". American documentation, v. 14, n. 1, pp. 10-25.

https://doi.org/10.1002/asi.5090140103

Leydesdorff, Loet (1991). "The static and dynamic analysis of network data using information theory". Social networks, v. 13 , n. 4, pp. 301-345.

https://doi.org/10.1016/0378-8733(91)90001-A

Leydesdorff, Loet; Wagner, Caroline S.; Bornmann, Lutz (2018). “Discontinuities in citation relations among journals: Self-organized criticality as a model of scientific revolutions and change". Scientometrics, v. 116, n. 1, pp. 623-644. https://doi.org/10.1007/s11192-018-2734-6

Tahamtan, Iman; Bornmann, Lutz (2018). “Creativity in science and the link to cited references: Is the creative potential of papers reflected in their cited references?". Journal of informetrics, v. 12, n. 3, pp. 906-930.

https://doi.org/10.1016/j.joi.2018.07.005

Van Eck, Nees-Jan; Waltman, Ludo (2014). "CitNetExplorer: A new software tool for analyzing and visualizing citation networks". Journal of informetrics, v. 8, n. 4, pp. 802-823.

https://doi.org/10.1016/j.joi.2014.07.006

Wu, Lingfei; Wang, Dashun; Evans, James A. (2019). “Large teams develop and small teams disrupt science and technology". Nature, v. 566, n. 7744, pp. 378-382.

https://doi.org/10.1038/s41586-019-0941-9

Wu, Shijie; Wu, Qiang (2019). "A confusing definition of disruption”. SocArxiv Papers.

https://osf.io/preprints/socarxiv/d3wpk

Bienvenido a 키

Revista cientifica internacional 